Check for updates

Cite this: Chem. Sci., 2018, 9, 8409

¿ All publication charges for this article have been paid for by the Royal Society of Chemistry

Received 1st June 2018

Accepted 6th September 2018

DOI: $10.1039 / c 8 s c 02415 d$

rsc.li/chemical-science

\title{
Branched BBB-shuttle peptides: chemoselective modification of proteins to enhance blood-brain barrier transport $\uparrow$
}

\author{
Cristina Díaz-Perlas, (D) ${ }^{a}$ Benjamí Oller-Salvia, (D) ${ }^{a}$ Macarena Sánchez-Navarro, (D) *a \\ Meritxell Teixidó (iD)*a and Ernest Giralt (iD *ab
}

\begin{abstract}
The blood-brain barrier (BBB) hampers the delivery of therapeutic proteins into the brain. BBB-shuttle peptides have been conjugated to therapeutic payloads to increase the permeability of these molecules. However, most BBB-shuttles have several limitations, such as a lack of resistance to proteases and low effectiveness in transporting large biomolecules. We have previously reported on the THRre peptide as a protease-resistant BBB-shuttle that is able to increase the transport of fluorophores and quantum dots in vivo. In this work, we have evaluated the capacity of linear and branched THRre to increase the permeability of proteins in cellular models of the BBB. With this purpose, we have covalently attached peptides with one or two copies of the BBB-shuttle to proteins in order to develop chemically welldefined peptide-protein conjugates. While THRre does not enhance the uptake and transport of a model protein in BBB cellular models, branched THRre peptides displaying two copies of the BBBshuttle result in a 2.6 -fold increase.
\end{abstract}

\section{Introduction}

Biological barriers hamper the efficient delivery of therapeutic molecules to their intended target and are thus one of the main challenges in contemporary medicinal chemistry. The bloodbrain barrier (BBB) is a physiological and metabolic barrier resulting from a complex interaction between endothelial cells and several other cell types, such as pericytes and astrocytes (Fig. 1). ${ }^{1-4}$ The protective nature of the BBB is conferred mainly by restrictive cell-to-cell connections called tight junctions. Disorders of the central nervous system (CNS), which include Alzheimer's and Parkinson's diseases, epilepsy, and brain cancers, have a high prevalence in modern society. There is therefore a need to develop new therapeutics to treat the aforementioned disorders. For those drugs that cannot cross the BBB unaided, the alternative is to conjugate them to molecules that do have this capacity and can thus transport them into the brain. ${ }^{5-7}$

Cell-penetrating peptides (CPPs) were developed to increase the intracellular delivery of drugs. ${ }^{\mathbf{8} 9}$ A large number of CPPs

${ }^{a}$ Institute for Research in Biomedicine (IRB Barcelona), Barcelona Institute of Science and Technology (BIST), Baldiri Reixac 10, Barcelona 08028, Spain. E-mail: macarena.sanchez@irbbarcelona.org; meritxell.teixido@irbbarcelona.org; ernest. giralt@irbbarcelona.org

${ }^{b}$ Department of Inorganic and Organic Chemistry, University of Barcelona, Marti $i$ Franquès 1-11, Barcelona 08028, Spain

† Electronic supplementary information (ESI) available. See DOI: $10.1039 / \mathrm{c} 8 \mathrm{sc} 02415 \mathrm{~d}$ have been described, including TAT, penetratin, SAP and octaarginine, for the delivery of a wide range of cargoes. ${ }^{\mathbf{1 0 , 1 1}}$ However, these molecules have several limitations, such as lack of selectivity or entrapment in endosomes. ${ }^{12}$ These issues can be tackled in a number of ways. ${ }^{11,13}$ On the one hand, the introduction of cyclic peptides opened the door to direct drug delivery to the cytosol. ${ }^{\mathbf{1 4}}$ On the other hand, CPP dendrimers display enhanced cell-penetrating properties. ${ }^{15}$ Reymond's

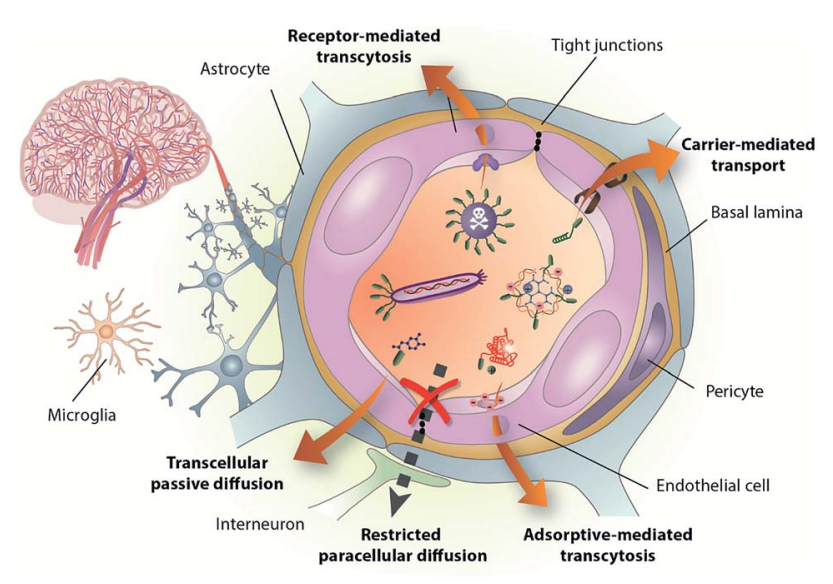

Fig. 1 The BBB is formed mainly by the tight brain endothelium, which is surrounded by the basal lamina and regulated by the other cells in the neurovascular unit, including pericytes, glial cells and neurons. BBB-shuttles mediate drug delivery across this barrier by taking advantage of endogenous transport pathways. Reproduced from ref. 1 with permission from the Royal Society of Chemistry. 
group reported a high multivalent effect for the divalent constructs of TAT, TP10 and Antp. ${ }^{16}$ Moreover, the branched dimerisation of the TAT peptide improved permeation potency in a non-linear manner. ${ }^{17}$

CPPs are not limited to intracellular delivery and have also been used to overcome other barriers, such as the gastrointestinal barrier and the BBB., ${ }^{\mathbf{1 0}, \mathbf{1 8}}$ Although CPPs can enter brain endothelial cells, recent findings show that the efflux rate of CPPs from the brain is higher than the influx in vivo. ${ }^{19}$ Due to their lack of selectivity and transcytosis, CPPs do not easily reach the brain parenchyma, thereby limiting their use as brain delivery vectors.

In recent years, a novel approach has been developed using molecular vectors, also known as Trojan horses or BBB-shuttles, to selectively deliver drugs into the CNS. ${ }^{20-22}$ One of the first peptides reported to overcome the BBB without disrupting it was Angiopep-2, currently the most advanced BBB-shuttle in clinical trials. ${ }^{2324}$ Angiopep-2 belongs to a first generation of BBB-shuttle peptides which also includes ApoE, ${ }^{25} \mathrm{THR}^{26}$ and TGN. ${ }^{27}$ The main flaw of this first generation was the lability to hydrolysis catalysed by peptidases, thus reducing their half-life in serum and their efficiency in vivo.

A second generation of BBB-shuttles was developed to overcome their stability limitations. ${ }^{28}$ These improvements included the cyclisation of peptides, ${ }^{29}$ the use of unnatural amino acids ${ }^{30}$ and the retro-enantio approach. These strategies have proven useful in the field of BBB-shuttle peptides, with examples such as THRre, ${ }^{31}{ }^{\mathrm{D}}$ Angiopep- $2{ }^{32}$ and ${ }^{\mathrm{D}} \mathrm{CDX} .{ }^{33}$

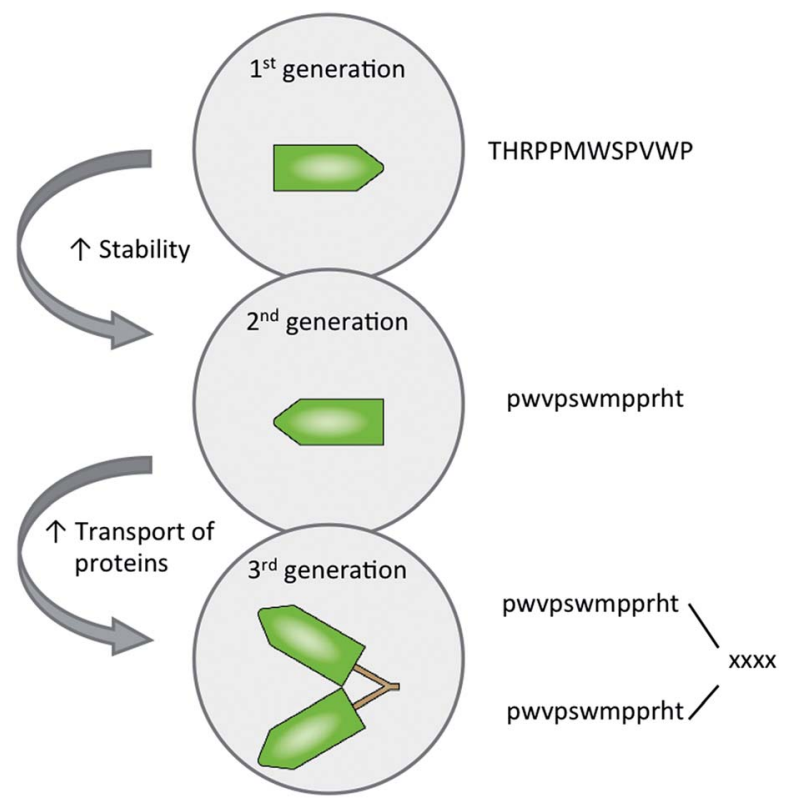

Fig. 2 Scheme representing three generations of BBB-shuttles. The $1^{\text {st }}$ generation is composed of peptides with L-amino acids that were first discovered to cross the BBB without disrupting it. The $2^{\text {nd }}$ generation improves their BBB-shuttle properties by conferring them with higher stability in front of serum proteases. In the $3^{\text {rd }}$ generation, the branched dimerisation of these BBB-shuttles improves the capacities of these peptides to transport more challenging cargoes. $x x x x$ represents the amino acids corresponding to the core of the branched peptide.
Here we present the design, synthesis and activity of a third generation of BBB-shuttles, namely branched BBB-shuttle peptides (Fig. 2). Although the second generation of BBBshuttles can find applications in the delivery of small molecules and inorganic nanoparticles (NPs), the real challenge is the delivery of proteins and antibodies to the brain. In the following sections, a branched version of the protease-stable peptide THRre is presented. In contrast to a single copy of the BBB-shuttle, the branched BBB-shuttle showed higher uptake and improved the permeability of a model protein in endothelial cells.

\section{Results and discussion}

\section{Synthesis of branched THRre peptides}

The BBB-shuttle THR (THRPPMWSPVWP) is a 12-mer peptide discovered by phage display that interacts with the human transferrin receptor. ${ }^{26}$ Although this peptide transports gold NPs across the BBB in vitro and in vivo, ${ }^{34}$ it has low stability to serum proteases. The retro-enantio version of THR (THRre, pwvpswmpprht) is stable in serum and is able to transport a variety of cargoes, with higher efficiency than the parent peptide, in an in vitro BBB cellular model and in vivo in mice. ${ }^{31}$

Branched peptides can be prepared in various ways. ${ }^{35}$ Lys has been used as the branching unit for its easy incorporation using solid-phase peptide synthesis (SPPS). Lys presents two amino functions, at the $\alpha$ - and $\varepsilon$-position, which provide two anchoring points. These Lys residues can either be intercalated in the main chain to create a decorated peptide ${ }^{36}$ or be placed at the C-terminus to form tree-like constructs. ${ }^{37,38}$

Multivalent peptides can be synthesised using stepwise SPPS or conjugation of two peptidic sequences forming the core and branches. Although the latter approach has the advantage of the pre-purification and characterisation of the separate peptides, the introduction of several chains into the core may be hampered by steric and hydrophobic interactions. ${ }^{38}$ Therefore, here we designed a stepwise synthesis of the branched THRre BBB-shuttle peptide. We synthesised the peptide with several

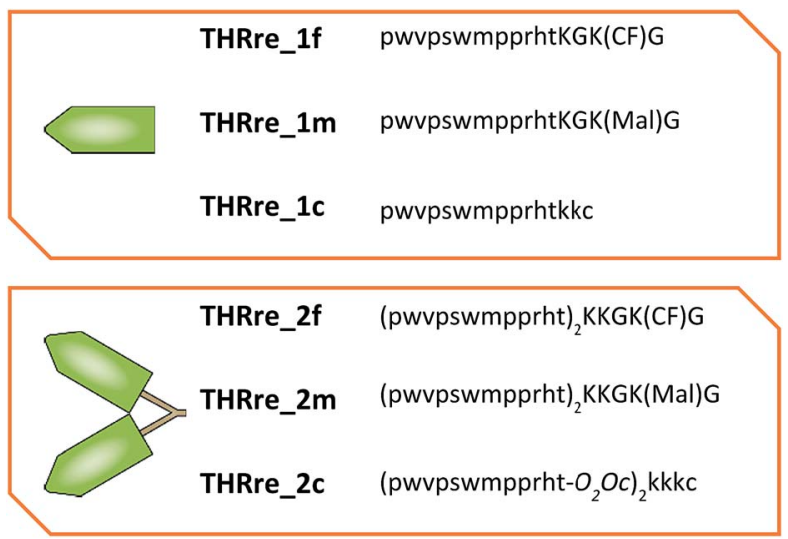

Fig. 3 Scheme representing the 6 peptides synthesised. Three versions of the peptide containing 1 copy and 2 copies of the THRre BBB-shuttle peptide were prepared with the amino acid sequences depicted. 
a
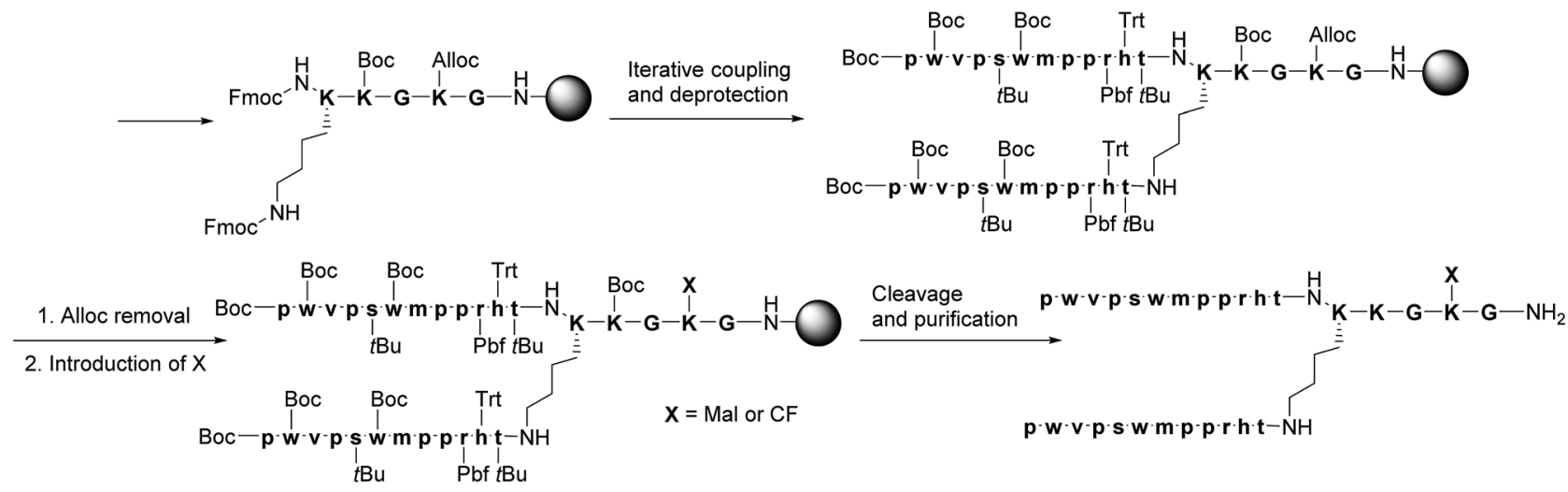

b
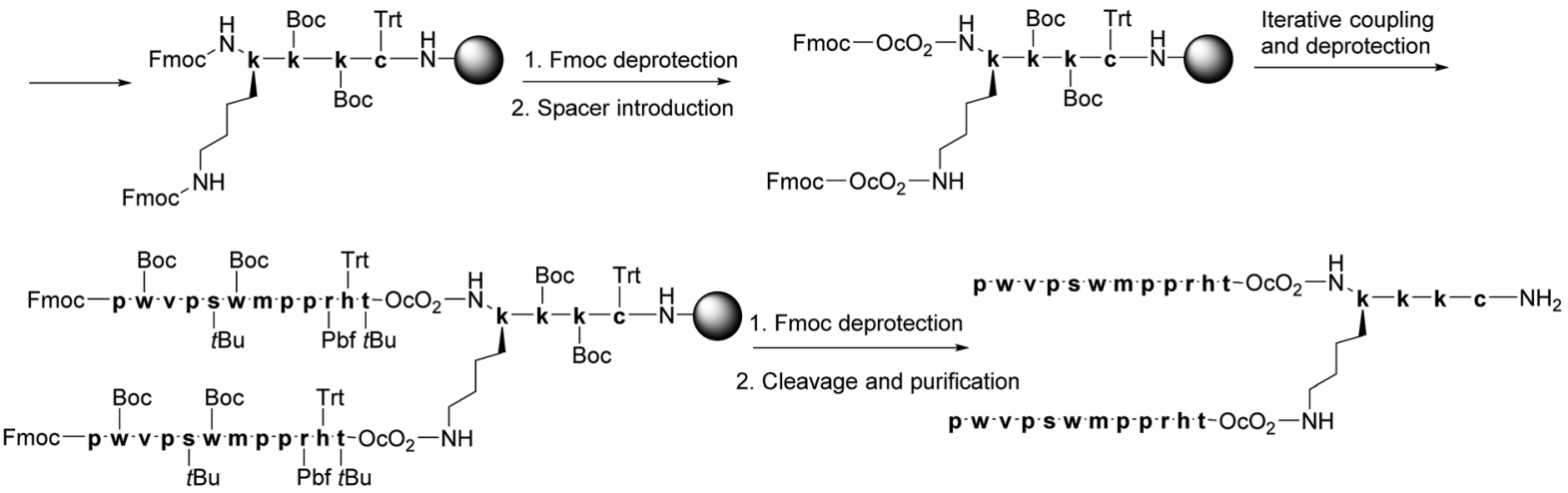

Scheme 1 Syntheses of the branched BBB-shuttle peptides. (a) Synthesis of THRre_2m ( $X=$ Mal) and THRre_2f ( $X=$ CF). The Alloc group was selectively removed before the cleavage to introduce other functional groups, such as 6-maleimidohexanoic acid (Mal) or carboxyfluorescein (CF). (b) Synthesis of THRre_2c.

modifications in order to facilitate the conjugation with proteins (Fig. 3 and Scheme 1). Fmoc-Lys(Fmoc)-OH was used as the branching unit, allowing the simultaneous deprotection of the two amino groups. A small spacer was introduced between the resin and the branching Lys in order to increase the solubility of the final peptides.

\section{Synthesis of THRre _2m and THRre _2f}

One of the Lys residues was orthogonally protected with an Alloc group to allow the introduction of other functionalities, such as a reactive moiety (6-maleimidohexanoic acid, Mal) or a fluorophore (carboxyfluorescein, CF) (Scheme 1a). Two Gly residues were placed in such a way as to flank the Lys(Alloc), thus serving as spacers. In this way, THRre_2m (with a Mal) and THRre_2c (with a CF) were obtained.

\section{Synthesis of THRre_2c}

An additional Cys residue was introduced at the C-terminus to provide a thiol as the reactive group (Scheme 1b). To improve the flexibility and solubility of the construct, the spacer 3,6dioxaoctanoic acid $\left(\mathrm{O}_{2} \mathrm{Oc}\right)$ was introduced between the branching Lys and the rest of the chain.

Similar to the branched BBB-shuttles, linear versions were also synthesised without the introduction of the branching unit thus presenting only one sequence of the BBB-shuttle sequence THRre (Fig. 3, S1 and Table S1†).

\section{Evaluation of the internalisation capacity in brain endothelial cells}

To evaluate the potential of the branched peptides as BBBshuttles, we examined their capacity to internalise a small cargo such as CF into cells. We assessed the amount of internalised peptides in a confluent monolayer of bEnd.3 cells, which is an immortalised mouse brain endothelial cell line that displays similar characteristics to the BBB. ${ }^{39}$ In these experiments, the peptides labelled with the fluorophore were incubated with the endothelial cells and, after a period of time, the amount of peptides internalised was measured. The results obtained by flow cytometry show a 20 -fold increase in uptake for the CF-labelled peptide containing two copies of THRre (THRre_2f) when compared with the one containing a single copy (THRre_1f) (Fig. 4a).

We then performed internalisation studies by confocal microscopy. These experiments were performed on bEnd.3 and also on hCMEC/D3 to show that the peptide is internalised both by murine and by human cells. ${ }^{40}$ Again, the amount of peptides internalised was higher for THRre_2f (Fig. 5, S3 and S4†).

In addition, THRre_2f was evaluated in a transcytosis BBB model that has a strong correlation with in vivo brain permeability ${ }^{41}$ This model consisted of human brain capillary endothelial cells, derived from pluripotent stem cells, forming a monolayer on the semi-permeable membrane of a transwell 
a \&

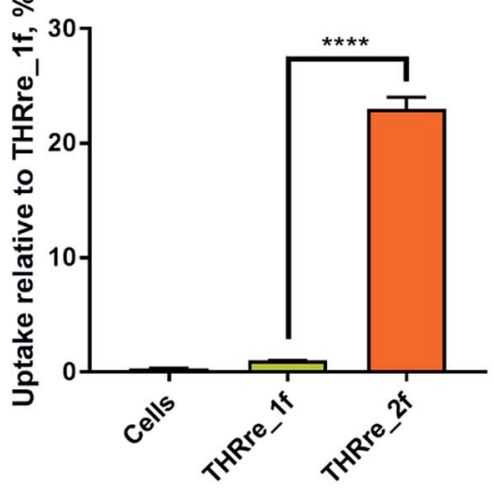

b

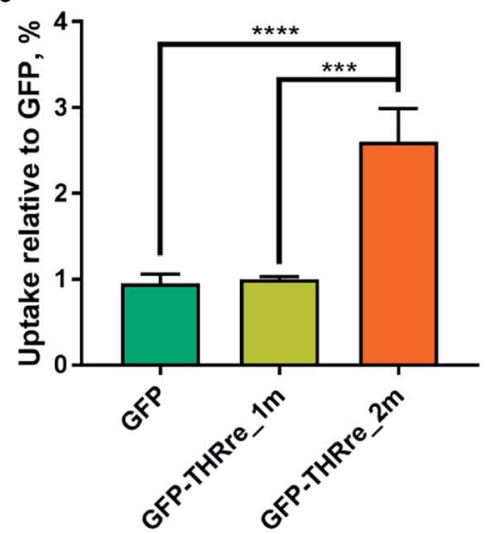

C

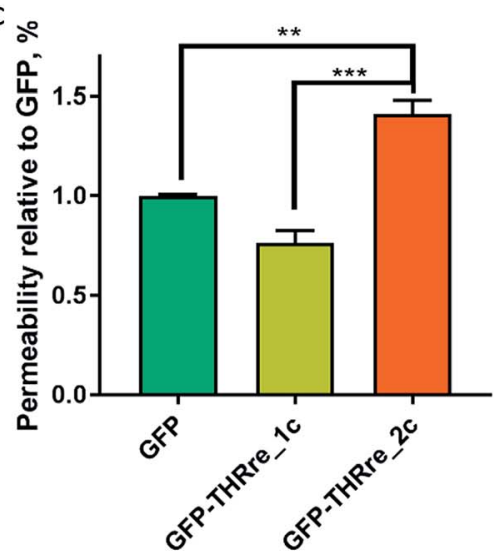

Fig. 4 (a) Uptake of the BBB-shuttles THRre_1f and THRre_2f by bEnd.3 cells. (b) Uptake of GFP conjugates by bEnd.3 cells. (c) Permeability in the in vitro BBB cellular model of GFP disulphide bridge conjugates. All values are reported as the mean \pm SEM $(n=3-10, * * * *<0.0001$, $* * * P<0.001, * * P<0.01 ; t$-test $)$

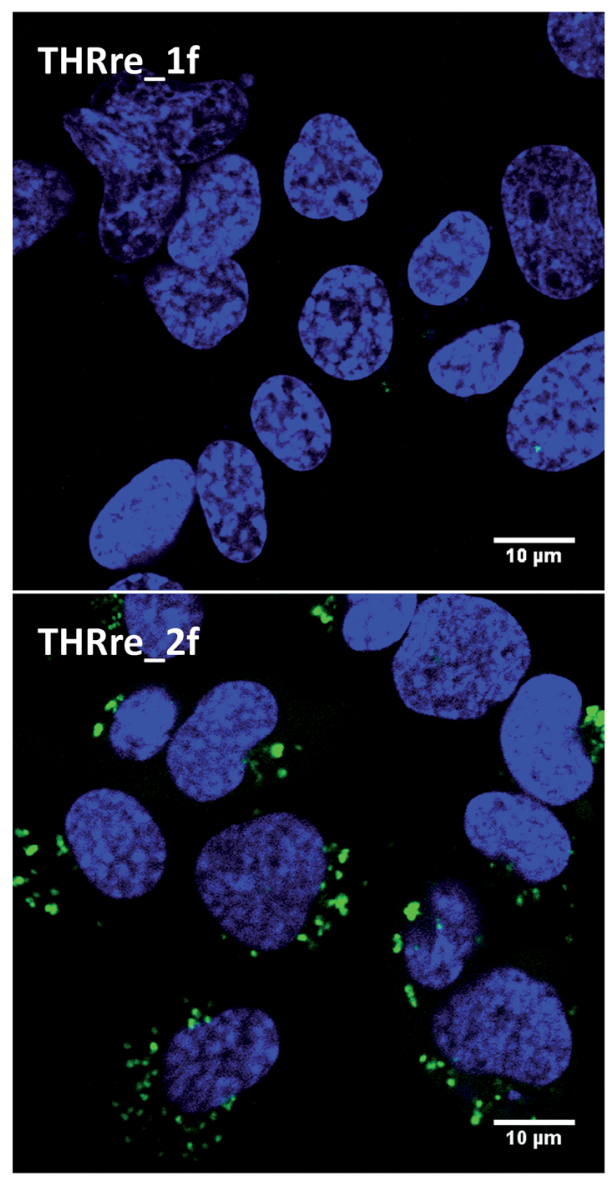

Fig. 5 Confocal fluorescence microscopy images of hCMEC/D3 cells after 30 min of incubation with THRre_1f or THRre_2f. CF-labeled peptides are shown in green and cell nuclei (Hoechst staining) in blue. The scale bar represents $10 \mu \mathrm{m}$.

and co-cultured with bovine pericytes. The apparent permeability measured was $(5.9 \pm 0.8) \times 10^{-6} \mathrm{~cm} \mathrm{~s}^{-1}$.

These results show that the presence of two copies of the BBBshuttle enhances the uptake of these peptides by endothelial cells. They also point towards a multivalent effect, where uptake is increased in a non-linear fashion with the number of copies of the peptide. Thus this platform could greatly facilitate the transport of proteins that are not able to reach the brain unaided.

\section{Chemoselective conjugation to model proteins}

Site-specific modification of proteins at a single residue is challenging and may not be enough to translocate the protein. Moreover, the introduction of several copies of a BBB-shuttle can provide an ill-defined mixture of chemical entities and may affect the physicochemical properties of the protein.

Branched peptides provide a chance to incorporate several BBB-shuttles at a single point, thus facilitating the conjugation, enhancing the BBB permeability and minimising impact on the protein properties and activity. The presence of more than one copy of the peptide in a nearby space would also increase the avidity of the peptide, resulting in a multivalent effect. ${ }^{17}$

Green fluorescent protein (GFP) was selected as a model for its ease of production and fluorescence properties. Moreover, GFP is not able to cross the BBB unaided, as is the case for most therapeutic proteins. ${ }^{42}$ The GFP used in these experiments was expressed with an extra Cys residue to allow selective conjugation to the BBB-shuttles. In this way, the conjugates will have a chemically well-defined structure, be easy to characterise and maintain the original activity of the protein (Fig. S5 $\dagger$ ).

Two types of conjugates were envisaged, either with a thioether linkage or a disulphide bridge (Scheme 2). The latter have a linkage that can be reduced in the cytosol and tumour environment, due to the presence of a high concentration of glutathione (GSH). Therefore, disulfide linked conjugates present interesting properties for the delivery of proteins into cells and to solid tumours since the proteins are released in their quasi-native form. ${ }^{43}$ To prove if GFP-THRre_1m and GFPTHRre_1c were released in the presence of intracellular levels of GSH and stable at blood levels of GSH the conjugates were incubated in a range of GSH concentrations (Fig. S7 $\dagger$ ). At $10 \mathrm{mM}$, only $50 \%$ of GFP-THRre_1c was detected after 20 


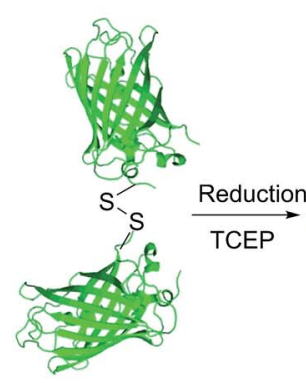

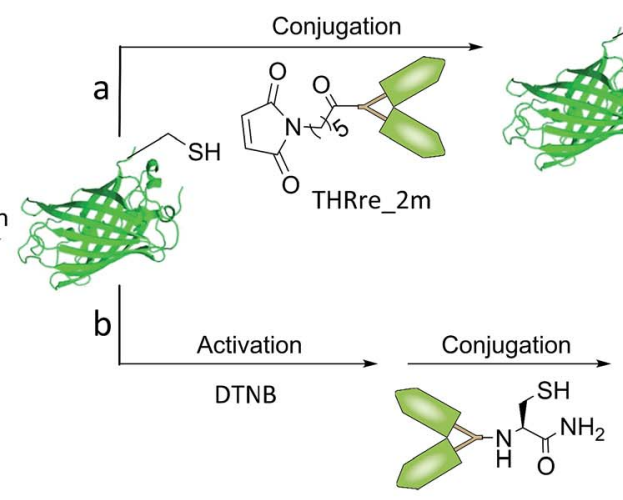

THRre 2c

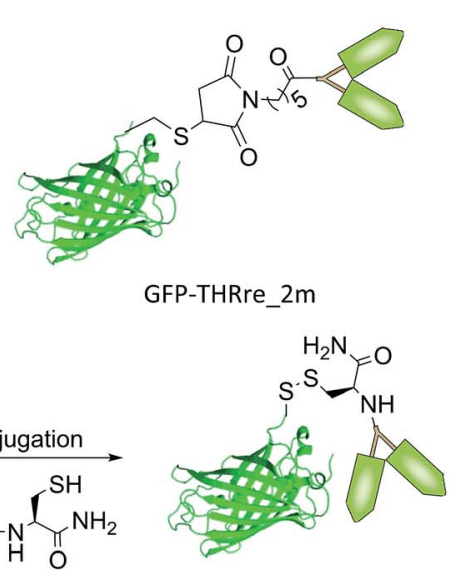

GFP-THRre_2c

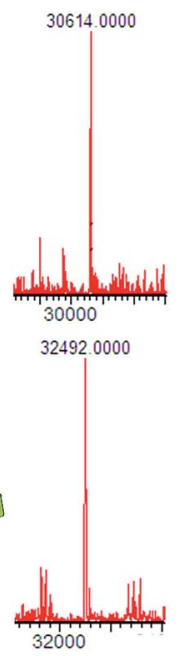

Scheme 2 Conjugation of the branched BBB-shuttle peptides to GFP via the formation of (a) a thioether linker or (b) a disulphide bridge. Spectra corresponding to the deconvoluted masses for the conjugates. For GFP-THRre_2m calc. MW $=32413 \mathrm{Da}$ and found MW $=32414 \mathrm{Da}$; and for GFP-THRre_2c calc. MW = $32496 \mathrm{Da}$ and found MW = $32492 \mathrm{Da}$. The same reactions were performed with the peptides containing one copy of the BBB-shuttle, obtaining GFP-THRre_1m and GFP-THRre_1c (Fig. S2 $\dagger$ ). DTNB = 5,5'-dithiobis-(2-nitrobenzoic acid).

minutes. By contrast, when the concentration of GSH was lowered to blood levels $(2 \mu \mathrm{M}), 100 \%$ of GFP-THRre_1c was intact after $1 \mathrm{~h}$.

\section{Conjugation via a thioether bridge}

Maleimide-containing peptides (THRre_1m and THRre_2m) were conjugated to GFP by the reaction of the maleimide moiety with the thiol of the Cys residue to form a stable thioether bridge. Following Scheme 2a, GFP was reduced by the addition of TCEP and conjugated without further purification with the maleimido-peptide to produce the conjugated product.

The conjugation of THRre_2m with the protein did not proceed to completion due to the bulkiness of this peptide construct in comparison with THRre_1m (Table S2, Fig. S2 and $\mathrm{S} 6 \dagger$ ).

\section{Conjugation via a disulphide bridge}

The disulphide bridge was achieved using the Cys-containing peptides (THRre_1c and THRre_2c) following Scheme 2b. The protein was reduced and reacted with Ellman's reagent to provide a more reactive intermediate thioester that reacted with the Cys-peptide to afford the final conjugated product. As in the previous conjugation, not all the protein reacted with the peptide in the constructs with THRre_2c (Table S2, Fig. S2 and $56 \dagger$ ).

In order to assess the importance of having a chemically well-defined structure, we attempted preparing a GFP conjugate with an average of two THRre molecules randomly distributed throughout the surface. However, all conditions tested (Fig. S8 and Table $\mathrm{S} 3 \dagger$ ) resulted in unstable conjugates. These results highlight the relevance of our strategy, which allows the incorporation of more than one BBB-shuttle molecule with a minimal effect on the protein properties.

\section{Evaluation of the BBB-shuttle properties of the conjugates}

After conjugation of the peptides to GFP, the BBB-shuttle properties of these constructs were evaluated through internalisation experiments in bEnd. 3 cells and transport across an in vitro human BBB cellular model. For these assays, the conjugates were labelled with ${ }^{125} \mathrm{I}$ so as to facilitate their detection. The radiolabelling allowed using a concentration of the conjugate in the range of those attained by therapeutic proteins in vivo. As negative control we used GFP that had been previously S-alkylated with iodoacetamide in order to prevent the thiol group from reacting in the cellular environment.

\section{Internalisation experiments}

The internalisation experiments were performed by incubating bEnd. 3 cells with the ${ }^{125}$ I-radiolabelled proteins for $30 \mathrm{~min}$. We assayed only the compounds with a thioether linkage because of its stability in a cellular environment. The uptake of GFP increased 2.6-fold when the protein was conjugated to THRre_2m while no increase was detected when conjugated to THRre_1m (Fig. 4b). These results are consistent with those obtained with CF as a cargo. The increase in uptake was exponential with the number of copies of the branched peptide, thereby suggesting that there is a synergistic effect between the peptides that enhances their BBB-shuttle properties.

\section{Permeability experiments}

Finally, an in vitro human BBB cellular model was used to further assess the BBB-shuttle properties of these conjugates.

In these experiments, the radiolabelled conjugates were placed in the donor compartment and, after a $2 \mathrm{~h}$ incubation, the amount that translocated across the endothelial cells and reached the acceptor compartment was measured. For these experiments, we used the conjugates with a disulphide bridge. 
The conjugates with THRre_2c (Papp $=(3.9 \pm 0.3) \times$ $10^{-6} \mathrm{~cm} \mathrm{~s}^{-1}$ ) had a higher Papp than the corresponding protein $\left((2.77 \pm 0.03) \times 10^{-6} \mathrm{~cm} \mathrm{~s}^{-1}\right)$ or conjugates with THRre_1c $\left((2.1 \pm 0.3) \times 10^{-6} \mathrm{~cm} \mathrm{~s}^{-1}\right)$ (Fig. $\left.4 \mathrm{c}\right)$. Of note, the permeability of the protein did not increase when only one copy of the BBBshuttle was present. These results are along the same lines as those obtained in the uptake experiments, thereby reinforcing the idea that two copies of the BBB-shuttle enhance the permeability of the protein. The high proximity of two THRre molecules in the branched structure makes simultaneous binding to more than one receptor highly improbable. Therefore, the increase in transport originated by the bivalent BBBshuttles should be attributed to an avidity effect. Two vicinal ligands result in an increased functional affinity due to a higher local concentration; ${ }^{\mathbf{4 4}}$ in the association-dissociation equilibrium established upon binding of one ligand, the close proximity of the other ligand further shifts the equilibrium toward binding.

\section{Conclusions}

Here we provide the first description of the design, synthesis and evaluation of branched BBB-shuttles to be used in the transport of chemically diverse cargoes, such as proteins. In this regard, branched BBB-shuttles were also conjugated to the model protein GFP. Peptide-protein conjugates containing two copies of the BBB-shuttle showed a non-linear increase in uptake. The evaluation in an in vitro human $\mathrm{BBB}$ cellular model confirmed that branched BBB-shuttles increase the permeability of GFP while only one copy cannot. Importantly, with this methodology, the conjugates have only one point modification, thereby allowing a well-defined chemical structure and the maintenance of the protein properties. This approach could be further applied to increase the efficiency of other BBB-shuttles to allow the delivery of larger and therapeutically relevant cargoes into the brain.

\section{Conflicts of interest}

There are no conflicts to declare.

\section{Acknowledgements}

This work was funded by MINECO-FEDER (BIO2016-75327-R), RecerCaixa-2014-Gate2Brain and Generalitat de Catalunya (XRB, 2017SGR0998 and 2016PROD00087 - this project has been financed with European Fund of Regional Development (FEDER)). We thank FEDAES/GENEFA and ASOGAF/BABEL FAMILY for support. IRB Barcelona is the recipient of a Severo Ochoa Award of Excellence from MINECO (Government of Spain) and the CERCA Programme of the Catalan Government. C. D.-P. holds a Predoctoral Grant Severo Ochoa (SVP-2013-067740).

\section{References}

1 B. Oller-Salvia, M. Sánchez-Navarro, E. Giralt and M. Teixidó, Chem. Soc. Rev., 2016, 45, 4690-4707.
2 N. J. Abbott, J. Inherited Metab. Dis., 2013, 36, 437-449.

3 W. M. Pardridge, Drug Discovery Today, 2007, 12, 54-61.

4 Y. Omidi and J. Barar, BioImpacts, 2012, 2, 5-22.

5 K. Nagpal, S. K. Singh and D. N. Mishra, Expert Opin. Drug Delivery, 2013, 10, 927-955.

6 W. M. Pardridge, Pharm. Res., 2007, 24, 1733-1744.

7 W. M. Pardridge, J. Cereb. Blood Flow Metab., 2012, 32, 19591972.

8 D. M. Copolovici, K. Langel, E. Eriste and Ü. Langel, ACS Nano, 2014, 8, 1972-1994.

9 G. Guidotti, L. Brambilla and D. Rossi, Trends Pharmacol. Sci., 2017, 38, 406-424.

10 M. Sánchez-Navarro, M. Teixidó and E. Giralt, Acc. Chem. Res., 2017, 50, 1847-1854.

11 D. Kalafatovic and E. Giralt, Molecules, 2017, 22, 1-38.

12 A. K. Varkouhi, M. Scholte, G. Storm and H. J. Haisma, J. Controlled Release, 2011, 151, 220-228.

13 M. Sánchez-Navarro, E. Giralt and M. Teixidó, Nat. Chem., 2017, 9, 727-728.

14 G. Lättig-Tünnemann, M. Prinz, D. Hoffmann, J. Behlke, C. Palm-Apergi, I. Morano, H. D. Herce and M. C. Cardoso, Nat. Commun., 2011, 2, 453.

15 G. A. Eggimann, E. Blattes, S. Buschor, R. Biswas, S. M. Kammer, T. Darbre and J.-L. Reymond, Chem. Commun., 2014, 50, 7254-7257.

16 G. A. Eggimann, S. Buschor, T. Darbre and J.-L. Reymond, Org. Biomol. Chem., 2013, 11, 6717.

17 I. A. Monreal, Q. Liu, K. Tyson, T. Bland, D. S. Dalisay, E. V. Adams, G. A. Wayman, H. C. Aguilar and J. P. Saludes, Chem. Commun., 2015, 51, 5463-5466.

18 M. Sánchez-Navarro, J. Garcia, E. Giralt and M. Teixidó, Adv. Drug Delivery Rev., 2016, 106, 355-366.

19 S. Stalmans, N. Bracke, E. Wynendaele, B. Gevaert, K. Peremans, C. Burvenich, I. Polis and B. De Spiegeleer, PLoS One, 2015, 10, 1-22.

20 W. M. Pardridge, Curr. Opin. Pharmacol., 2006, 6, 494-500.

21 M. Malakoutikhah, M. Teixidó and E. Giralt, Angew. Chem., Int. Ed., 2011, 50, 7998-8014.

22 A. G. de Boer and P. J. Gaillard, Annu. Rev. Pharmacol. Toxicol., 2007, 47, 323-355.

23 M. Demeule, J. C. Currie, Y. Bertrand, C. Ché, T. Nguyen, A. Régina, R. Gabathuler, J. P. Castaigne and R. Béliveau, J. Neurochem., 2008, 106, 1534-1544.

24 A. Régina, M. Demeule, C. Ché, I. Lavallée, J. Poirier, R. Gabathuler, R. Béliveau and J. P. Castaigne, Br. J. Pharmacol., 2008, 155, 185-197.

25 D. Wang, S. S. El-Amouri, M. Dai, C.-Y. Kuan, D. Y. Hui, R. O. Brady and D. Pan, Proc. Natl. Acad. Sci. U. S. A., 2013, 110, 2999-3004.

26 J. H. Lee, J. A. Engler, J. F. Collawn and B. A. Moore, Eur. J. Biochem., 2001, 268, 2004-2012.

27 J. Li, L. Feng, L. Fan, Y. Zha, L. Guo, Q. Zhang, J. Chen, Z. Pang, Y. Wang, X. Jiang, V. C. Yang and L. Wen, Biomaterials, 2011, 32, 4943-4950.

28 S. Lien and H. B. Lowman, Trends Biotechnol., 2003, 21, 556-562. 29 B. Oller-Salvia, M. Sánchez-Navarro, S. Ciudad, M. Guiu, P. Arranz-Gibert, C. Garcia, R. R. Gomis, R. Cecchelli, 
J. García, E. Giralt and M. Teixidó, Angew. Chem., Int. Ed., 2016, 55, 572-575.

30 P. Arranz-Gibert, B. Guixer, M. Malakoutikhah, M. Muttenthaler, F. Guzman, M. Teixido and E. Giralt, J. Am. Chem. Soc., 2015, 137, 7357-7364.

31 R. Prades, B. Oller-Salvia, S. M. Schwarzmaier, J. Selva, M. Moros, M. Balbi, V. Grazú, J. M. De La Fuente, G. Egea, N. Plesnila, M. Teixidó and E. Giralt, Angew. Chem., Int. Ed., 2015, 54, 3967-3972.

32 X. Wei, C. Zhan, X. Chen, J. Hou, C. Xie and W. Lu, Mol. Pharm., 2014, 11, 3261-3268.

33 X. Wei, C. Zhan, Q. Shen, W. Fu, C. Xie, J. Gao, C. Peng, P. Zheng and W. Lu, Angew. Chem., Int. Ed., 2015, 54, 3023-3027.

34 R. Prades, S. Guerrero, E. Araya, C. Molina, E. Salas, E. Zurita, J. Selva, G. Egea, C. López-Iglesias, M. Teixidó, M. J. Kogan and E. Giralt, Biomaterials, 2012, 33, 7194-7205.

35 S. H. Medina and M. E. H. El-Sayed, Chem. Rev., 2009, 109, 3141-3157.
36 G. Kragol and L. Otvos, Tetrahedron, 2001, 57, 957-966.

37 W. Kowalczyk, B. G. De La Torre and D. Andreu, Bioconjugate Chem., 2010, 21, 102-110.

38 W. Kowalczyk, M. Monsó, B. G. de la Torre and D. Andreu, J. Pept. Sci., 2011, 17, 247-251.

39 R. C. Brown, A. P. Morris and R. G. O'Neil, Brain Res., 2007, 1130, 17-30.

40 B. Weksler, I. A. Romero and P.-O. Couraud, Fluids Barriers CNS, 2013, 10, 16.

41 R. Cecchelli, S. Aday, E. Sevin, C. Almeida, M. Culot, L. Dehouck, C. Coisne, B. Engelhardt, M. P. Dehouck and L. Ferreira, PLoS One, 2014, 9, e99733.

42 W. M. Pardridge, BioDrugs, 2017, 31, 503-519.

43 H. D. Herce, D. Schumacher, A. F. L. Schneider, A. K. Ludwig, F. A. Mann, M. Fillies, M. A. Kasper, S. Reinke, E. Krause, H. Leonhardt, M. C. Cardoso and C. P. R. Hackenberger, Nat. Chem., 2017, 9, 762-771.

44 G. Vauquelin and S. J. Charlton, Br. J. Pharmacol., 2013, 168, 1771-1785. 